María A. Borrueco Rosa

borrueco@us.es

Universidad de Sevilla

(Recibido: 1 junio 2019/ Received: $1^{\text {st }}$ June 2019) (Aceptado: 15 octubre 2019/ Accepted: $15^{\text {th }}$ October 2019)

\section{APORTACIONES DE LA NEUROCIENCIA COGNITIVA \\ AL APRENDIZAJE DE LA L2 EN \\ EL AULA INFANTIL. ESTUDIO APLICADO AL ALEMÁN COMO LENGUA EXTRANJERA}

\author{
CONTRIBUTIONS OFCOGNITIVE SCIENCE TO CHILD \\ L2 LEARNING. A STUDY APPLIED TO GERMAN AS A \\ FOREIGN LANGUAGE
}

\title{
Resumen
}

Las aportaciones de la neurociencia al conocimiento de los procesos de aprendizaje han generado una corriente cada vez más extendida de investigaciones que consideran los avances en el funcionamiento cerebral como fundamentales para el diseño de recursos metodológicos en el aula. La neuroeducación como ámbito interdisciplinar que aúna conocimientos neurocognitivos y didácticos para el desarrollo de dinámicas en el aula infantil, se presenta como el espacio idóneo para integrar aspectos nuevos como el funcionamiento de la memoria, los procesos atencionales o las emociones, que hasta el momento estaban excluidos de la didactización de procesos de enseñanzaaprendizaje. La neurociencia viene a demostrar de forma clara cuestiones ya asentadas desde la perspectiva didáctica y materializadas en recursos como las metodologías activas o el aprendizaje colaborativo. Aún se han de integrar aspectos endógenos al aprendizaje como la relevancia de los procesos emocionales en la percepción y procesamiento de los estímulos para que se produzca de forma efectiva el aprendizaje. El presente estudio recoge algunas de las aportaciones en torno a los fundamentos del aprendizaje desde la perspectiva neurocognitiva y plantea como objetivo considerarlos en la didáctica del alemán como lengua extranjera en el aula infantil.

Palabras clave: neurociencia educativa, neurodidáctica, neuroeducación, L2 infantil, alemán como lengua extranjera.

\section{Abstract}

The contributions of neuroscience to the knowledge of learning processes have generated a wide range of researches. These works consider the new evidences on brain functioning as major factors when dealing with the design of methodological resources for the classroom. Neuroeducation is an interdisciplinary area that combines neurocognitive and didactic knowledge and put them at the service of the better development of educational dynamics aimed to children. This area is therefore presented as the proper frame to join new aspects such as the operational system of the memory, attention processes or emotions, which so far have been excluded from the systematization and description of the teaching-learning process. Neuroscience comes to unambiguously prove some issues, already assumed by the Didactics and already transformed into resources like active methodologies or 
collaborative learning. Yet some aspects attached to Learning as a discipline are still to be integrated, i.e., emotional processes and stimulus processing have been proved to be relevant factors when it comes to success in learning. The present study hosts some contributions to the principles of Learning from a neurocognitive approach and sets as an objective the inclusion of these contributions within the frame of the teaching-learning process of German as a foreign language in children.

Key words: educational neuroscience, neurodidactics, neuroeducation, child L2 learning, German as a foreign language

\section{Introducción}

Desde la perspectiva didáctica y metodológica los estudios en torno al aprendizaje de una lengua extranjera han centrado su atención generalmente en la lengua misma (Sánchez, 2009) y desde principios de siglo en los recursos metodológicos que optimizan el proceso de adquisición de destrezas (Díaz-Corralejo, 2003). Las metodologias activas en el aula y el desarrollo de competencias, las subcompetencias necesarias y las destrezas resultantes como vías de comunicación en la lengua extranjera, han sido y siguen siendo objeto de investigación tanto a nivel teórico como experimental en el aula. Generan en la actualidad numerosos proyectos de innovación docente y estudios derivados que suelen fundamentarse en resultados cuyo valor se cuantifica según aspectos conductuales observables en el aula. Estos se evalúan en cuanto a la definición de la competencia comunicativa según el Marco Común Europeo de Referencia para las Lenguas (MCERL) y el consiguiente desarrollo de destrezas de comprensión y expresión oral y escrita (Figueras, 2005).

En este contexto irrumpe la neurociencia como fuente de información para pedagogos y docentes (Wolfe, 2001; Barrera y Donolo, 2009), generando ámbitos de investigación interdisciplinares aún confusos pero que poco a poco van delimitando objetivos concretos (Ansari, Coch y De Smedt, 2010). Asi disciplinas ya reconocidas como la neuroeducación (Mora, 2013; Salcedo, 2018) o la neurodidáctica (Roth, 2011) van acercando posiciones en cuanto a objetivos de investigación comunes y que impulsan iniciativas dirigidas hacia lo que algunos autores denominan "la neurociencia educativa":

The scientists at National Brain Research Centre in consort with like-minded researchers have initiated work in this direction under the aegis of the Cognitive Science Programme of DST. To provide an impetus for such collaboration and its wider dissemination, we propose to bring this activity under the umbrella of 'Educational Neuroscience' (Chatterjee, 2016: 65).

Proponemos la posibilidad de poner al servicio del docente datos sobre el cerebro relevantes para el proceso de aprendizaje de la lengua extranjera en el aula (Borrueco y Alberca, 2018). Hasta qué punto la neurociencia es relevante para enseñar, no deja de ser una cuestión controvertida (Bowers, 2016). No son pocos los que rechazan la incursión en aspectos neurocientíficos como recurso para modular las estrategias docentes en el aula (Terigi, 2016), postulando la existencia de neuromitos en torno a las capacidades cerebrales (Papatzikis, 2017) o cuestionando la proliferación de estudios teóricos que no aportan soluciones efectivas en el aula y son generados a partir de teorías científicas reduccionistas (Terigi, 2016).

A falta de estudios aplicados, numerosas aportaciones en torno a la neurociencia y educación se reducen a la defensa de postulados generales que, a pesar de su interés, se manifiestan como insuficientes para extraer consecuencias concluyentes en cada materia objeto de aprendizaje. Como 
iniciativa pionera podemos citar los estudios realizados por Cantó (2015) en torno a la educación infantil.

En este contexto, nos postulamos a favor de aportaciones interdisciplinares que planteen como metodología de investigación la estrecha interacción entre la neurociencia y la materia especifica objeto de aprendizaje. A este binomio añadimos el marco en el que se define el perfil del aprendiente, que en el contexto que nos ocupa, será determinante. Los espacios que confluyen en este estudio son pues, por un lado, los estudios neurocognitivos sobre procesos cerebrales implicados en el proceso de aprendizaje y, por otro, las características específicas que definen el sistema lingüístico alemán. Nos limitaremos a desarrollar aspectos concretos que definen el aprendizaje de la L2 desde ambas perspectivas en el aula infantil.

La metodología de trabajo parte de la observación de carencias del proceso de aprendizaje de la lengua alemana como L2 como lengua extranjera aplicables al aula infantil y de la determinación de posibles soluciones mediante propuestas didácticas experimentadas en el aula que faciliten el aprendizaje adecuado al cerebro. Para ello se realizará una revisión bibliográfica de trabajos que evidencian el proceso de aprendizaje desde el punto de vista neurocognitivo, por un lado, y de recursos didácticos que facilitan los procesos atencionales necesarios para fomentar el desarrollo de destrezas, por otro.

\section{Planteamiento del problema: Diagnóstico y carencias del proceso de aprendizaje de alemán como lengua extranjera (DaF) en el aula infantil}

Desde la perspectiva didáctica actual se establece en las aulas de lengua extranjera, independientemente del perfil del alumno, como objetivo preferente la competencia comunicativa (Núñez y Moral, 2010), entendida esta como un compendio de subcompetencias según el MCERL que han de ser desarrolladas para generar las cuatro destrezas tradicionales de comprensión y expresión oral y escrita. Hasta el momento el proceso de didactización se ha limitado a la selección de partes de la lengua para ser didactizadas en actividades al servicio de la progresión lingüística de la L2.

Observamos en esta descripción del perfil de enseñanza/aprendizaje de lenguas extranjeras actual, por un lado, la concentración de elementos exógenos al procesamiento cognitivo. No detectamos motivación ontogénica que considere los aspectos neurocognitivos inherentes al proceso de aprendizaje y aún menos, reflejos del proceso de aprendizaje endógeno al desarrollo cognitivo del niño.

Se plantea la duda de si estos métodos no son más que una adaptación de los correspondientes a métodos de aprendizaje para adultos, con objeto de simplificar el proceso de aprendizaje y facilitar así la adquisición de la L2 al niño, en nuestro caso la lengua alemana. De forma análoga al método adulto, los estímulos presentados, léxico y gramática contextualizadas en situaciones comunicativas, consideran la programación de objetivos bajo un criterio de progresión cíclica según niveles de dificultad lingüística. Generalmente esta dificultad viene determinada por las reglas gramaticales de la lengua alemana en un entorno léxico de situaciones comunicativas muy semejantes a las situaciones comunicativas diseñadas para adultos. Solo en algunos contextos de aprendizaje se consideran actividades que motiven al niño a participar activamente en la construcción de conocimientos. Estas carencias se manifiestan sobre todo a nivel metodológico en el aula. En numerosos contextos de aprendizaje infantil el docente actúa siguiendo el principio de isomorfismo pedagógico y transmite estrategias de aprendizaje propias, que reproducen situaciones de aprendizaje instructivistas experimentadas en la edad adulta y que conllevan estrategias inadecuadas, como puede ser la adquisición explícita de reglas gramaticales, su memorización y aplicación en ejercicios de reproducción. En el 
contexto de la lengua alemana, la dificultad de algunos aspectos centrales como la declinación, tanto en entornos nominales como de adjetivos, dificultades inherentes al sistema verbal alemán como la formación de participios o la voz pasiva o la hipotaxis, todos de características muy divergentes a la lengua materna, concluyen en entornos de aprendizaje que impulsan claramente metodologias conductistas en las que se impone la imitación y reproducción de modelos sin producirse aprendizaje significativo, lo que genera un alto grado de frustración en el aprendiz y el abandono del interés por el aprendizaje efectivo de la lengua alemana. En estos, además, se adquiere de forma implícita el vocabulario y se reproducen en situaciones dirigidas que impiden la comunicación auténtica en el aula.

Nos planteamos la posibilidad de superar el modelo tradicional fundamentado en la progresión lingüistica, para determinar otros parámetros como punto de partida de la programación docente y que estos sean seleccionados de forma coherente al desarrollo cognitivo del niño, sus motivaciones, sus habilidades y su entorno socio-afectivo.

\section{Aportaciones de la neurociencia al proceso de aprendizaje de la L2 en el aula infantil}

Chatterjee (2016: 64) refleja claramente la influencia bidireccional entre neurociencia y educación:

There are 2 main streams of knowledge which link neuroscience to education: (i) brain structures responsible for various educational processes like reading, attention, memory, calculation, language acquisition, etc. and (ii) the manner in which educational processes affect brain structure and function.

Si desde la perspectiva conductista el aprendizaje se concibe como un cambio duradero en el potencial conductual y para el cognitivismo supone principalmente cambios en las asociaciones (Ertmer y Newby, 1993; García 2012), los estudios neurocientíficos determinan que el aprendizaje está relacionado con los cambios que se producen a nivel neuronal, cognitivo $y$, en consecuencia, conductual, como resultado de una experiencia y con objeto de adaptarse al entorno. Cambiamos una conducta especifica en relación a la experiencia previa y a los estímulos presentes (Klein, 2003).

La teoría conexionista (Ledesma, 2015) defiende que el conocimiento no deriva de la adquisición de reglas abstractas sino de la activación recurrente de información y del anclaje de esta información mediante conexiones neuronales. La investigación en neurociencia cognitiva evidencia de forma concluyente un cambio en la arquitectura sináptica cuando se produce un determinado aprendizaje.

Para muchos autores el nivel de desarrollo del lenguaje humano, es, además de una de las principales caracteristicas que nos diferencian de los animales, la base de otras peculiaridades y singularidades comportamentales y cognitivas. Más allá de la discusión científica acerca de la adquisición del lenguaje en nuestra especie, y en el individuo joven, si es una capacidad innata, o si es adquirida, como defiende la vertiente funcionalista, lo que es claro es que la adquisición plena de la capacidad lingüistica del humano tiene lugar en la edad infantil (Sánchez-Reyes, 2000). En lo que sí hay diferencia de opiniones es en la definición del nivel de competencia que se llega a adquirir en un momento u otro del desarrollo ontogénico y en la diferencia de esta influencia según la dimensión lingüística de la que se trate. 
Desde la perspectiva neurocognitiva destacamos dos aspectos de interés para nuestro estudio. Por un lado, el concepto de memoria y los factores que determinan su desarrollo (Ullman, 2004), y, por otro, aspectos específicos del desarrollo neurocognitivo del niño que pudiesen ser de interés para nuestra propuesta didáctica de DaF en el aula infantil.

\subsection{El aprendizaje desde la perspectiva neurocognitiva: Ios procesos atencionales, las emociones, y la memoria}

La atención, entendida en los términos que definen Álvarez, González-Castro, Núñez, González-Pienda, Álvarez y Bernardo (2007) como el procedimiento directamente implicado en la recepción activa de la información, está relacionada tanto con procesos cognitivos como motivacionales. La atención se entiende como un mecanismo que conecta procesos cognitivos con afectivos, y como un factor determinante para decidir qué estímulos serán analizados de forma prioritaria.

Este modelo neoconexionista se complementa con el modelo de recursos limitados de Kanheman (1973) para quien la atención no solo depende de la disponibilidad para atender del individuo, sino también de las exigencias de la tarea a desarrollar. La atención selectiva que discrimina estímulos dentro de conjuntos y la atención sostenida, relacionada con la capacidad de concentración (Buschman y Kastner, 2015), configuran asi parte de los procesos atencionales determinantes para que la información sea procesada. No hemos de olvidar, por otra parte, que en los procesos atencionales juega un papel muy importante el nivel de automatismo como señala Bermejo (1987). El niño, a medida que va automatizando procesos cognitivos, va liberando carga cognitiva para desarrollar otras habilidades.

En este proceso atencional complejo, el cerebro ignora lo ya conocido, diferencia lo importante de lo irrelevante, construye categorias, modelos y jerarquías, ordena acontecimientos en secuencias lógicas y finalmente integra lo nuevo en lo ya conocido (Textor, 2010). Así todo lo que es novedoso produce nuevas sinapsis, y todo lo reiterado crea hábitos por repetición, estabilizándose las conexiones (Paniagua, 2016).

Estas conexiones participan en el proceso de memorización de información (Schacter y Tulving, 1994), conformando según la función desempeñada distintos tipos de memoria. Ruiz-Contreras y Cansino (2015) definen el funcionamiento de la memoria como la sucesión de una fase de codificación y adquisición de la información y una fase posterior de recuperación. Es de máximo interés para nuestro estudio constatar que la memoria sensorial alberga información con alta carga emocional: "La información tiene tinte emocional asignado por la amígdala. Si lo sensorial es significativo para el sujeto, pasa a las áreas de asociación que elaboran conocimientos, pensamientos concretos o abstractos o ideas, que están impregnadas de emoción". (Paniagua, 2016: 89).

La memoria a corto plazo, de unos segundos, se sustenta en las experiencias, y la información que alberga, si es repetida, pasará a conformar la memoria a largo plazo (Axmachar, Haupt, Cohen, Elger y Fell, 2009). La información, si se "encuentra debidamente organizada, codificada y repetida, no se pierde" (Paniagua, 2016: 90). Según el tipo de información, podrá ser considerada episódica, declarativa o semántica y procedimental (Eichenbaum y Neal, 2001; Schacter y Tulving, 1994; Tulving, 1985). Conocer la diferencia entre ellas resulta de máximo interés para el docente de la L2, pues pueden contribuir a la optimización de la conectividad neuronal y de esta forma al desarrollo de competencias. Además, conocer el funcionamiento especifico de la memoria infantil, nos ayudará a comprender su evolución en la adquisición de la lengua extranjera.

Panigua (2016) destaca la memoria episódica como característica de la etapa infantil. El desarrollo preferente de esta memoria en el niño, de código temporal y espacial, hace que recuerde de for- 
ma preferente los hechos del dia y no los conceptos ni los procesos. La constatación de esta realidad debería ser un elemento determinante para la programación de actividades en el aula. Frente al código temporal y espacial de la memoria episódica, la memoria semántica tiene código verbal y la procedimental, código motor. Si la primera alberga items de información contextual, la segunda almacena procesos y secuencias, dando lugar al pensamiento organizado y a la automatización de habilidades, y entre otras, posibilita la habilidad para mantener la atención (Paniagua, 2016). La conjunción de ambas memorias será determinante para la automatización de los procesos lingüísticos necesarios para el desarrollo de la comunicación en la L2 (Garcia, 2012; Ullman, 2004).

Damasio (1998) y Mora (2013) destacan en sus estudios neurocientíficos que todo aprendizaje, es decir, toda modificación de la estructura neuronal (Kandel y Hawkins, 1992), y, por tanto, la memoria, tiene contenido emocional (Ruiz-Contreras y Cansino, 2015). La emoción y la motivación influyen en el nivel atencional del individuo, y por tanto en el filtro que decide qué información se atenderá y aprenderá y cuál se ignorará (Rueda, Posner y Rothbart, 2005). La influencia de las emociones en el desarrollo cognitivo e intelectual del ser humano es tal que se ha acuñado el término de inteligencia emocional (Goleman, 1996).

Otros autores, además de las teorías estimulares, hablan de una evolución en la capacidad perceptiva como el factor decisivo a la hora de discriminar información. Este tipo de atención dicótica o selectiva es de especial relevancia para nuestro estudio, pues como apunta Bermejo (1987), los cambios de edad implican cambios en los procesos atencionales y, por lo tanto, requieren de un análisis específico según el desarrollo neurocognitivo. Así, los niños pequeños son más susceptibles a la distracción y no discriminan fácilmente información irrelevante. Con el incremento de la edad, aumenta, por ejemplo, el nivel de atención a la información relevante.

En este mismo sentido se manifiestan Marina (2014) y Paniagua (2016), que defienden que, junto al aprendizaje cognitivo imbuido de carga emocional, no se produce aprendizaje si no se desarrollan determinadas capacidades ejecutivas como fijar objetivos y regular a voluntad nuestra conducta. El marcador de inteligencia ejecutiva se muestra cuando se es capaz de inhibir estímulos externos, evitar distracciones y mantener enfocada la atención en una sola tarea para elaborar metas.

Según lo expuesto hasta el momento, consideramos desde la perspectiva didáctica, que la naturaleza del estímulo y, sobre todo, la forma en la que presentamos la secuencia de estímulos (Morgan-Short, Steinhauer, Sanz y Ullman, 2012), junto con los procesos meta-atencionales de autocontrol voluntario destacados por Bermejo (1987), conformarian los factores determinantes en el proceso de percepción.

\subsection{A prendizaje de lenguas extranjeras y cronobiología del desarrollo infantil}

Lo expuesto hasta el momento nos permite deducir que el aprendizaje de la lengua extranjera, como cualquier otro proceso de aprendizaje, está directamente relacionado con el desarrollo neurocognitivo del sujeto. Aprendemos a hablar una lengua, materna o una L2 porque el uso de la lengua genera huellas en el cerebro (Alberca y Borrueco, 2018; Costa y Sebastián-Gallés, 2014).

Si entendemos el aprendizaje como modificación de la arquitectura sináptica, es interesante considerar que se constata en el niño de edad temprana una actividad muy superior de neurogénesis. La optimización de la eficiencia en las conexiones entre esas neuronas (pruna neuronal) que se da durante la infancia posibilita el aprendizaje a altas velocidades de comportamientos, lengua, estilo de vida, y de cuanto rodea al niño, permitiendo que permanezcan solo los enlaces estables (Johnson y Newport, 1989). Es lo que posibilita el conocido como periodo crítico de aprendizaje de lenguas extranjeras: 
A modo de ejemplo, la fase sensible tiene una duración que se prolonga hasta los seis o siete años de vida. El bebé es capaz de diferenciar todos los sonidos de cada una de las lenguas del mundo y el niño es capaz de generar todos los fonemas correctamente. Pocos años después se eliminan todas las sinapsis que posibilitan esta capacidad, pero ya no son necesarias dado que el niño adquiere por lo general una única lengua con un número limitado de fonemas (Textor, 2010).

Durante el periodo crítico la experiencia tiene fuertes efectos en el desarrollo cerebral y en el comportamiento. Pasado este período los estímulos necesarios para producir cambios cerebrales han de ser mayores. En el caso concreto de la adquisición de una segunda lengua, sabemos que el individuo, pasado su período crítico puede aprender una L2, pero con mayor dificultad (Kuhl, 2010). Se plantea la duda de hasta qué punto influye la edad de adquisición en el dominio de una L2 y en qué aspectos particulares, cuestión que ha planteado no pocas controversias (Torres, 2005).

Parece que los niños que aprenden una L2 en edad temprana lo hacen de igual manera que su L1, es decir, de forma implícita, incidental y automática. Digamos que aprenden lenguas, la materna 0 la L2, mientras están aprendiendo las bases del lenguaje, y en este período solo por la exposición a los estímulos, por la propia experiencia y de un modo implícito no consciente (bottom-up), el individuo joven aprende los fonemas y la gramática. Lanauze y Snow (1989) encontraron que había una transferencia de las capacidades y habilidades de la L1 a la L2, incluso antes de que las competencias orales y auditivas en la $L 2$ estuvieran plenamente desarrolladas.

A partir de este momento el cerebro optimiza el proceso de memoria: las conexiones neuronales que son importantes permanecen, las irrelevantes son eliminadas. La configuración neuronal que ha desarrollado hasta el momento favorecerá el desarrollo de habilidades posteriores. Así el niño bilingüe aprenderá con mayor facilidad una tercera o cuarta lengua (Sánchez-Reyes, 2000).

Los estudios apuntan a que esta ventana de tiempo supuestamente determinante para el aprendizaje de una lengua concierne sobre todo a los aspectos fonológicos. Al nacer, el ser humano es capaz de responder a todos los fonemas existentes con la misma intensidad de respuesta, y aprender a reproducirlos con la misma facilidad. Es durante sus primeros años que el humano se vuelve especialista de su entorno, por la importancia adaptativa al mismo, y por tanto, especialista del idioma que se hable en él. Así, para el desarrollo de la percepción auditiva, durante los primeros 6 meses de edad un bebé tiene una capacidad absoluta para cualquier idioma (Kuhl, 2010) y es a partir de los 6 a 12 meses cuando empieza a especializarse en los fonemas de su lengua materna (Kuhl, Stevens, Hayashi, Deguchi, Kiritani e Iverson, 2006). Estos fonemas se estabilizan y pueden actuar como un filtro perceptivo que dificulte el procesamiento de los fonemas de la L2 si estos son muy distintos a los de la L1.

De igual forma, se registran estudios que apuntan a que la edad de adquisición de la gramática también influye en su nivel de dominio. Johnson y Newport (1989) constataron un incremento en los errores al aplicar diferentes reglas gramaticales del inglés conforme más tarde comenzaron los sujetos a adquirir esa $L 2$.

En el contexto del aprendizaje de una L2 que nos ocupa, es interesante destacar que la adquisición del vocabulario en el niño es lenta, pues es paralela a su proceso madurativo. La interacción con el medio es fundamental, pues este aporta imágenes sensoriales del vocabulario que va aprendiendo. Estas imágenes darán lugar a conceptos que permitirán que relate hechos de su vida, generando aprendizaje semántico con múltiples conexiones neuronales (Paniagua, 2016).

\footnotetext{
1 Traducción propia.
} 
Es destacable para nuestro propósito mencionar que el aprendizaje por imitación es de gran relevancia. El niño elabora durante la imitación una planificación motora en su mente que hace posible enviar órdenes directas al organismo, generando aprendizajes sin error y por instrucción directa (Paniagua, 2016).

Es interesante, además, resaltar que el juego, recurso preferente en la infancia, genera gran cantidad de endorfinas que reducen el nivel de estrés (Roth, 2011). Mediante el juego desarrolla y ejecuta movimientos, adquiere nociones de espacio, procesos lógicos y toma decisiones. Estos aprendizajes construirán otros aprendizajes complejos.

Concluimos este apartado mencionando algunos aspectos relevantes para el aprendizaje de lenguas a partir de los estudios de Textor (2010) y Paniagua (2016):

1-3 años: Se produce la explosión lingüistica tras el desarrollo cognitivo correspondiente.

3-4 años: Se desarrolla la memoria a largo plazo.

4-7 años: Se desarrolla la capacidad analítica, diferencia entre imaginado y realidad, y es capaz de adoptar roles.

6-12 años: Su creciente madurez cerebral le permite desarrollar el pensamiento lógico, y establecer metas de forma autónoma. A partir de los 7 años el niño aprende si así lo desea, el aprendizaje se ha convertido en un acto de voluntad.

+ 12 años: Se observa la adquisición de la inteligencia ejecutiva, pues ya ha desarrollado freno inhibitorio, verbal y motor, elabora y mantiene metas.

\section{El aprendizaje del alemán en el aula infantil: perspectiva neurodidáctica}

Si la consideración de la neurociencia educativa (Chatterjee, 2016) conlleva la integración de consecuencias didácticas en el modelo tradicional de aprendizaje, proponemos optimizar el modelo existente, partiendo del concepto de competencia comunicativa (Fig. 1) según el MCERL (2003)

Generalmente, este paradigma de competencias implícitas en las cuatro destrezas comunicativas se desarrolla según la pedagogía moderna en un entorno de recursos de aprendizaje por tareas, cooperativo y mediante metodologías activas (Fernández March, 2006). Aúna esta descripción del paradigma docente actual, teoría y método de aprendizaje (Fernández, 2009), postulando desde el constructivismo pedagógico (Reich, 1998) la experiencia como fuente de desarrollo de destrezas y situando al aprendiz en el centro del paradigma, otorgándole papel de actante principal y generador de sus competencias. La neurociencia viene así a confirmar supuestos pedagógicos reconocidos, aunque en la práctica aún no son aplicados de forma generalizada.

Las consecuencias didácticas derivadas de las aportaciones sobre la naturaleza del aprendizaje desde la perspectiva neurocognitiva y algunos aspectos centrales en torno al desarrollo neurocognitivo del niño pueden concretarse en los siguientes aspectos que relacionamos a continuación apoyándonos en las teorias de aprendizaje de Bransford, Brown y Cooking (1999); Textor (2010) y Paniagua (2016), entre otros. Proponemos un modelo de aprendizaje de la L2 que considere de un lado los objetivos comunicativos (Fig. 1) y, por otro, las recomendaciones derivadas del aprendizaje adecuado al cerebro (Fig. 2). 


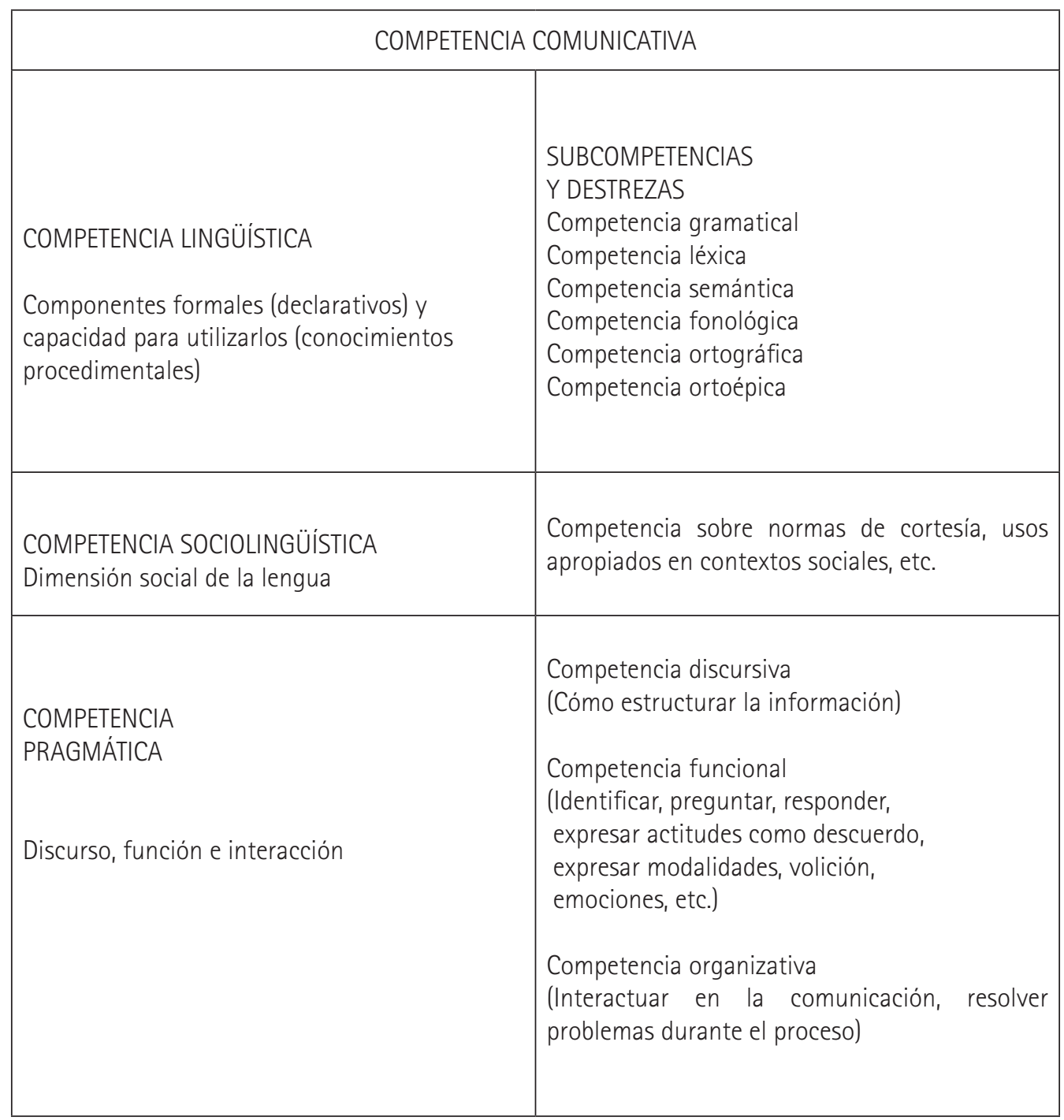

(Fig. 1). Competencia comunicativa (Elaboración propia).

Para aplicar este modelo en al aula es necesario que el docente sea consciente de la oportunidad y necesidad de intervenir y actuar sobre el método y la programación establecida, pues ha de adaptarse a la progresión real del grupo de aprendientes y a su respuesta a los estímulos de la L2. Es responsabilidad del docente responder con curiosidad y respeto a las reacciones del niño en el aula, formulando respuestas de recompensa positiva, limitando en lo necesario la corrección del error, pues puede ser potenciado si recibe excesiva atención. Se ha constatado que las emociones se manifiestan como fundamentales en la generación de conocimientos, que las metas a modo de desafíos y retos impulsan el aprendizaje y que el miedo lo bloquea (Mora, 2013). 
Modelo de aprendizaje según la neurociencia educativa FACTORES DETERMINANTES

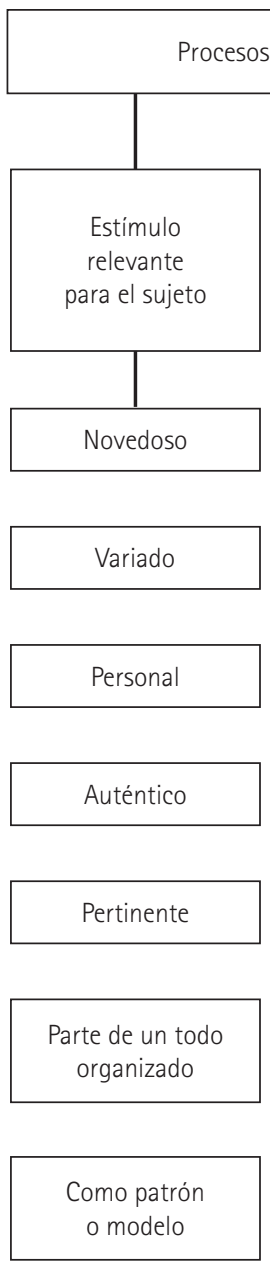

Como medio para potenciar la seguridad en el niño
Estímulos adecuados a la madurez del sujeto en procesos analíticos

Estímulos adecuados
para potenciar la
atención sostenida y
la concentración en la
consecución de una
meta

Estímulos adecuados para potenciar la atención selectiva discriminando estímulos dentro de un conjunto
Procesos de cambio en la estructura neuronal

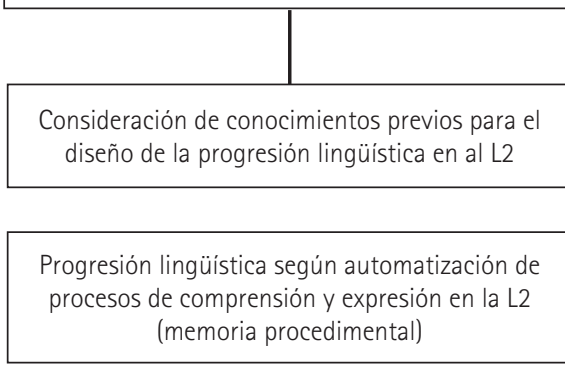

Procesos de asociación mediante la experiencia adecuados a la madurez neurocognitiva del sujeto

Estímulos secuenciados según la madurez neurocognitiva en cuanto a aprendizaje implícito y explícito de la L2

\section{suleto}

Procesos de anclaje de la información
potenciando la memoria sensorial con
recursos de relevancia emocional para elaborar
pensamientos impregnados de emoción
positiva

Procesos de recuperación de la memoria mediante feed back constante y progresión cíclica de actualización de conocimientos previos (conexiones neuronales)

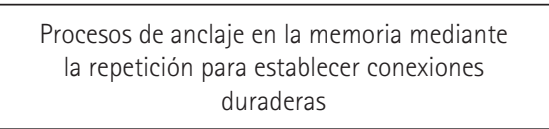

Procesos de aprendizaje explícito para potenciar la memoria declarativa y procedimental de forma simultánea al aprendizaje implicito para la automatización de procesos

Procesos de anclaje en la memoria mediante entornos auténticos para establecer conexiones duraderas relevantes en la memoria episódica

Fig. 2. Modelo de factores determinantes en neurociencia educativa (Elaboración propia). 
Ante la imposibilidad de presentar un estudio global por niveles, nos limitaremos a hacer algunas propuestas que ilustren la conjunción de factores analizados hasta el momento. En este sentido es interesante mencionar iniciativas como la propuesta por Álvarez et alii, (2007: 211) que proponen la creación de "bancos de actividades secuenciadas" y ejercicios con variantes según los objetivos planteados y una evaluación diagnóstica del grupo de aprendientes. Los bancos de actividades serían un modelo de material didáctico abierto a la evolución cognitiva y lingüistica del grupo.

Los bancos de actividades estarían a disposición del equipo docente, por ejemplo, para desarrollar la atención selectiva identificando estímulos sonoros o visuales, comparando estímulos en cuanto a sus cualidades, identificando estímulos dentro de series, reconociendo estímulos en el espacio, entre otros. De igual forma serian beneficiosos bancos de actividades para desarrollar la atención sostenida con objeto de reproducir modelos, iguales u opuestos, reconstruir modelos según un orden establecido, visualizar grupos de estímulos léxicos, sonoros o visuales o bancos de juegos adecuados al perfil del niño: sensoriales, motores, socio-emocionales y cognoscitivos y abstractos (Álvarez et alii, 2007). Quedarían definidos según los objetivos lingüísticos y se desarrollarían según las reglas preestablecidas del juego.

Es posible encontrar en red una gran variedad de ejercicios muy interesantes, adecuados a la metodología en el aula infantil, pero en su mayoria fundamentados en el recurso de la lectoescritura, con lo que no cumplimos con la recomendación de presentar el estímulo considerando los procesos atencionales o de construcción de redes neuronales mencionados anteriormente. Las asociaciones entre significado y grafía son representadas mediante iconos y el grafema correspondiente y acompañados de ejercicios de aplicación o reconstrucción de significados, reproduciendo así el modelo de aprendizaje adulto.

Veamos algunas propuestas alternativas que describimos según los parámetros de la neurociencia educativa analizados anteriormente. Entendemos que van dirigidos a docentes de la lengua extranjera en el aula infantil, por lo que el objetivo es describir las situaciones de aprendizaje relacionadas con la lengua. Los parámetros que consideramos son, de una parte, la activación de procesos atencionales, y, de otra, la organización del material lingüístico de acuerdo a criterios neurocognitivos. Las propuestas se fundamentan en las fuentes mencionadas a lo largo del estudio.

Se presenta a continuación el esbozo de algunos bancos de actividades diseñados con objeto de crear entornos de aprendizaje adecuados al cerebro en el aula infantil de alemán como lengua extranjera.

\subsection{Entorno multimodal}

Este entorno motivaría una programación motivacional de partida que sería asociada posteriormente a la programación lingüística, y debería sustentarse en competencias motoras. Adquiere importancia la relación entre la actividad y el objetivo comunicativo. Veamos un ejemplo de programación multimodal variada en la que la progresión viene determinada por la relevancia del estímulo variado y vinculado al concepto de embodiment o enacción (Varela, Thompson y Rosch, 1991):

\begin{tabular}{|c|l|l|l|l|l|l|l|l|}
\hline malen & Singen & Ausschneiden & spielen & basteln & schreiben & dramatisieren & sich bewegen & anmalen \\
\hline
\end{tabular}

Fig. 3 Progresión motivacional (Elaboración propia) 
A este conjunto multimodal de acciones es posible asociar objetivos comunicativos y destrezas, y así conectar actividades motoras de integración somato-sensorial con situaciones comunicativas adecuadas:

\begin{tabular}{|c|c|}
\hline Situación comunicativa & $\begin{array}{l}\text { Programación motivacional } \\
\text { (Procesos atencionales) }\end{array}$ \\
\hline $\begin{array}{l}\text { Mein Klassenraum } \\
\text { Destrezas: Expresión oral / } \\
\text { Comprensión oral } \\
\text { Competencia léxica y gramatical }\end{array}$ & $\begin{array}{l}\text { Tarjetas de acción para posibilitar el desplazamiento por el aula } \\
\text { Buscar objetos en el aula } \\
\text { Situar según órdenes objetos en un lugar } \\
\text { Buscar objetos ocultos }\end{array}$ \\
\hline Familie und Freunde & $\begin{array}{l}\text { Regalar un dibujo a un amigo del grupo } \\
\text { Representación teatral asumiendo roles familiares }\end{array}$ \\
\hline Kleidung & Elaborar disfraces / disfrazarse \\
\hline Meine Spielzeuge & $\begin{array}{l}\text { Traer un juguete al aula y nombrarlo } \\
\text { Juego libre en el aula / describir acciones jugando }\end{array}$ \\
\hline Wir spielen Einkaufen & Juego de rol / Escenificación de diálogos \\
\hline Wir lernen Tiere kennen & $\begin{array}{l}\text { Visionado de videos educativos sobre animales y representar / } \\
\text { reconocer animales } \\
\text { Reconocer animales por onomatopeya }\end{array}$ \\
\hline
\end{tabular}

Fig. 4. Situación comunicativa y programación motivacional.

A estas actividades delimitadas por la relevancia del estímulo quedan vinculadas según las intenciones comunicativas programadas, los objetivos lingüisticos, tanto fonológicos, léxicos, morfosintácticos, ortográficos y ortoépicos de la lengua alemana, es decir, integramos en objetivos lingüísticos, objetivos cognitivos, sociales y emocionales potenciando la creatividad del niño: 


\begin{tabular}{|c|c|c|c|}
\hline Situación comunicativa & $\begin{array}{l}\text { Programación motivacional } \\
\text { (Procesos atencionales) }\end{array}$ & $\begin{array}{l}\text { Objetivo lingüístico } \\
\text { (determinados según progresión } \\
\text { lingüística) }\end{array}$ & $\begin{array}{l}\text { Criterios } \\
\text { neurocognitivos }\end{array}$ \\
\hline $\begin{array}{l}\text { Mein Klassenraum } \\
\text { Destrezas: Expresión } \\
\text { oral / } \\
\text { Comprensión oral } \\
\text { Competencia léxica y } \\
\text { gramatical }\end{array}$ & $\begin{array}{l}\text { Tarjetas de acción para } \\
\text { posibilitar el desplazamiento } \\
\text { por el aula. } \\
\text { Buscar objetos en el aula. } \\
\text { Situar según órdenes objetos } \\
\text { en un lugar. } \\
\text { Buscar objetos ocultos. }\end{array}$ & $\begin{array}{l}\text { Sustantivos y géneros } \\
\text { Preposiciones locales } \\
\text { auf / unter / neben / vor / hinten } \\
\text { Verbos con desplazamiento en el } \\
\text { espacio; aufstehen sich setzen, } \\
\text { stellen, verstecken, suchen, ... }\end{array}$ & $\begin{array}{l}\text { Conocimientos previos } \\
\text { Automatización } \\
\text { Asociación mediante } \\
\text { experiencia } \\
\text { Memoria sensorial } \\
\text { Aprendizaje implícito }\end{array}$ \\
\hline $\begin{array}{l}\text { Familie und Freunde } \\
\text { Destrezas: Expresión } \\
\text { oral / } \\
\text { Comprensión oral } \\
\text { Competencia léxica y } \\
\text { gramatical }\end{array}$ & $\begin{array}{l}\text { Regalar un dibujo a un amigo } \\
\text { del grupo. } \\
\text { Realizar un dibujo de la } \\
\text { familia o amigos que han de } \\
\text { describir al colgar en un panel } \\
\text { del aula. } \\
\text { Representación teatral } \\
\text { asumiendo roles familiares. }\end{array}$ & $\begin{array}{l}\text { Sustantivos y géneros } \\
\text { Conjugación verbal. } \\
\text { Posesivos. } \\
\text { Oración afirmativa, interrogativa, } \\
\text { negativa. } \\
\text { Números. } \\
\text { Singular y plural. }\end{array}$ & $\begin{array}{l}\text { Memoria episódica } \\
\text { Entorno auténtico } \\
\text { Relevancia emocional } \\
\text { Asociación mediante } \\
\text { experiencia }\end{array}$ \\
\hline $\begin{array}{l}\text { Kleidung } \\
\text { Competencia léxica y } \\
\text { fonética. }\end{array}$ & $\begin{array}{l}\text { Elaborar disfraces y } \\
\text { disfrazarse. }\end{array}$ & $\begin{array}{l}\text { Sustantivos y colores para } \\
\text { describir disfraces y prendas. }\end{array}$ & $\begin{array}{l}\text { Asociación mediante la } \\
\text { experiencia } \\
\text { Aprendizaje implícito }\end{array}$ \\
\hline $\begin{array}{l}\text { Meine Spielzeuge } \\
\text { Competencia léxica y } \\
\text { morfosintáctica }\end{array}$ & $\begin{array}{l}\text { Traer un juguete al aula y } \\
\text { nombrarlo } \\
\text { Juego libre en el aula y } \\
\text { describir acciones jugando }\end{array}$ & $\begin{array}{l}\text { Sustantivos y géneros. } \\
\text { Conjugación verbal en torno a } \\
\text { acciones relacionadas con el juego }\end{array}$ & $\begin{array}{l}\text { Asociación mediante la } \\
\text { experiencia } \\
\text { Recuperación de la } \\
\text { memoria mediante } \\
\text { contextos auténticos }\end{array}$ \\
\hline $\begin{array}{l}\text { Wir lernen Tiere } \\
\text { kennen } \\
\text { Comprensión oral } \\
\text { Expresión oral }\end{array}$ & $\begin{array}{l}\text { Video sobre animales } \\
\text { Reconocer y describir } \\
\text { animales por onomatopeya } \\
\text { e imágenes Personalizar las } \\
\text { acciones }\end{array}$ & $\begin{array}{l}\text { Corpus léxico de animales } \\
\text { Verbos de acción relacionados } \\
\text { Conjugación en singular / plural }\end{array}$ & $\begin{array}{l}\text { Memoria declarativa } \\
\text { Aprendizaje implícito }\end{array}$ \\
\hline $\begin{array}{l}\text { Wir spielen Einkaufen } \\
\text { Competencia lingüistica, } \\
\text { sociolingüística y } \\
\text { pragmática }\end{array}$ & $\begin{array}{l}\text { Juego de rol. } \\
\text { Dramatización de situaciones } \\
\text { comunicativas }\end{array}$ & $\begin{array}{l}\text { Flexión nominal } \\
\text { Verbos modales } \\
\text { Estructura oracional }\end{array}$ & $\begin{array}{l}\text { Anclaje lingüistico } \\
\text { mediante entornos } \\
\text { auténticos por asunción } \\
\text { de rol }\end{array}$ \\
\hline
\end{tabular}

Fig. 5. Programación según criterios neurocognitivos / motivacionales. 


\subsection{Entornos de desarrollo cognitivo}

El objetivo en este tipo de bancos de actividades es el desarrollo cognitivo encaminado a desarrollar el lexicón mental tanto nominal como verbal y los conocimientos asociados a ellos.

\begin{tabular}{|c|c|c|c|}
\hline Situación comunicativa & $\begin{array}{l}\text { Programación motivacional } \\
\text { (Procesos atencionales) }\end{array}$ & $\begin{array}{l}\text { Objetivo lingüístico } \\
\text { (determinados según } \\
\text { progresión lingüística) }\end{array}$ & Criterios neurocognitivos \\
\hline $\begin{array}{l}\text { Essen und Trinken } \\
\text { Competencia léxica } \\
\text { y lectoescritura } \\
\text { Competencia ortográfica } \\
\text { Competencia ortoépica }\end{array}$ & $\begin{array}{l}\text { Recortar imágenes de } \\
\text { comidas y bebidas de folletos } \\
\text { de supermercado y clasificar } \\
\text { según categorias de fruta, } \\
\text { verdura, carnes, pescado, } \\
\text { bebidas, ... } \\
\text { Seleccionar de todos los } \\
\text { recortes uno y formar grupos } \\
\text { por categorias en el aula. }\end{array}$ & $\begin{array}{l}\text { Sustantivos y géneros } \\
\text { Adjetivos } \\
\text { Colores } \\
\text { Sinónimos / antónimos } \\
\text { Hiperónimos } \\
\text { Adverbios de cantidad } \\
\text { Números }\end{array}$ & $\begin{array}{l}\text { Memoria declarativa por } \\
\text { repetición } \\
\text { Discriminación de } \\
\text { información } \\
\text { Elaboración de patrones } \\
\text { Desarrollar la atención } \\
\text { selectiva y sostenida }\end{array}$ \\
\hline Mein Klassenraum & $\begin{array}{l}\text { Rol auténtico docente- } \\
\text { alumno: seleccionar entre } \\
\text { el material escolar el objeto } \\
\text { nombrado. }\end{array}$ & Sustantivos y géneros & $\begin{array}{l}\text { Discriminación fonética y } \\
\text { semántica }\end{array}$ \\
\hline Farben lernen & $\begin{array}{l}\text { Proyección de imágenes para } \\
\text { asociar colores coherentes } \\
\text { a la realidad mar - azul / } \\
\text { naranja - fruta/color / fuego } \\
\text { - amarillo / hoja - verde. } \\
\text { Nombrar los colores de } \\
\text { objetos personales una vez } \\
\text { adquirido el dominio sobre } \\
\text { palabras en alemán del } \\
\text { conjunto de colores. }\end{array}$ & $\begin{array}{l}\text { Sustantivos y géneros. } \\
\text { Adjetivos. }\end{array}$ & $\begin{array}{l}\text { Atención selectiva } \\
\text { Discriminación fonética de } \\
\text { elementos dentro de un } \\
\text { conjunto } \\
\text { Recuperación de la } \\
\text { memoria mediante acceso } \\
\text { semántico inducido. } \\
\text { Desarrollo de procesos } \\
\text { analíticos. }\end{array}$ \\
\hline Freizeitaktivitäten & $\begin{array}{l}\text { Clasificar tarjetas bilingües } \\
\text { según actividades en } \\
\text { entornos diferentes (casa / } \\
\text { parque / campo/ playa ) }\end{array}$ & $\begin{array}{l}\text { Categorización semántica de } \\
\text { verbos }\end{array}$ & $\begin{array}{l}\text { Categorización a partir } \\
\text { de un proceso de cotejo } \\
\text { de características de lo } \\
\text { percibido, identificación } \\
\text { y determinación de } \\
\text { similitudes y diferencias. }\end{array}$ \\
\hline $\begin{array}{l}\text { Körperteile } \\
\text { Competencia léxica }\end{array}$ & $\begin{array}{l}\text { Dibujar, recortar partes del } \\
\text { cuerpo y reconstruir de forma } \\
\text { colectiva un cuerpo humano. } \\
\text { Nombrar habilidades según } \\
\text { las partes del cuerpo }\end{array}$ & $\begin{array}{l}\text { Sustantivos y géneros } \\
\text { Competencia semántica verbal. }\end{array}$ & $\begin{array}{l}\text { Integración del todo y sus } \\
\text { partes } \\
\text { Anclaje semántico } \\
\text { mediante } \\
\text { contextualización }\end{array}$ \\
\hline
\end{tabular}

Fig. 6. Programación según criterios de desarrollo cognitivo. 


\subsection{Entornos de interacción social}

El objetivo en este tipo de bancos de actividades es posibilitar espacios de trabajo colaborativo, grupales y en situaciones comunicativas afines al individuo. El ser humano es un animal social que necesita de los otros para su supervivencia. De ahí la necesidad de entender nuestro entorno y a nuestros congéneres. El origen de la empatía se puede relacionar con esta necesidad de adaptación al medio social. El aprendizaje vicario o por imitación es uno de los ejemplos de esta vinculación con los otros individuos, y algunos autores identifican las "neuronas espejo" como su caso más extremo (Rizzolati, 2005).

\begin{tabular}{|c|c|c|c|}
\hline Situación comunicativa & $\begin{array}{l}\text { Programación motivacional } \\
\text { (Procesos atencionales) }\end{array}$ & $\begin{array}{l}\text { Objetivo lingüístico } \\
\text { (determinados según } \\
\text { progresión lingüística) }\end{array}$ & Criterios neurocognitivos \\
\hline $\begin{array}{l}\text { Competencia léxica y } \\
\text { verbal en torno a los } \\
\text { conceptos temporales de } \\
\text { tiempo. }\end{array}$ & $\begin{array}{l}\text { Exposición ante el grupo de las } \\
\text { estaciones del año (primavera, } \\
\text { verano, otoño, invierno) } \\
\text { mediante elaboración de un } \\
\text { mural representativo de las } \\
\text { cuatro estaciones describiéndolas } \\
\text { según fiestas, tiempo atmosférico } \\
\text { y acciones cotidianas asociadas } \\
\text { a ellos }\end{array}$ & $\begin{array}{l}\text { Corpus léxico de estaciones, } \\
\text { meses, tiempo atmosférico, } \\
\text { fiestas, acciones cotidianas. } \\
\text { Sustantivos y géneros } \\
\text { Verbos de acción } \\
\text { Sistema preposicional } \\
\text { temporal } \\
\text { Flexión nominal de } \\
\text { sustantivo } \\
\text { Conjugación verbal } \\
\text { impersonal y personal en } \\
\text { singular y plural. }\end{array}$ & $\begin{array}{l}\text { Trabajo en grupo con } \\
\text { funciones delimitadas } \\
\text { para potenciar el trabajo } \\
\text { colaborativo y coordinado } \\
\text { Aprendizaje "lernen durch } \\
\text { lehren". }\end{array}$ \\
\hline Expresión oral y escrita & $\begin{array}{l}\text { En un folleto de productos de } \\
\text { un supermercado alemán, se } \\
\text { ha de seleccionar un grupo de } \\
\text { alimentos con un presupuesto } \\
\text { de dinero dado en el que se ha } \\
\text { de ajustar la cantidad de dinero } \\
\text { en Euros a las cantidades o } \\
\text { continentes según corresponda } \\
\text { a gramos, kilos, botes, piezas, } \\
\text { botella. } \\
\text { Se ha de elaborar un diálogo que } \\
\text { se representará escenificado en } \\
\text { grupo, en el que se seleccionan } \\
\text { los alimentos a lo largo del } \\
\text { supermercado y se abonan en } \\
\text { caja. }\end{array}$ & $\begin{array}{l}\text { Adverbios de cantidad. } \\
\text { Conjuntos y unidades de } \\
\text { alimentos. } \\
\text { Continentes y contenidos } \\
\text { contables e incontables. } \\
\text { Formulación de preguntas y } \\
\text { respuestas, afirmaciones y } \\
\text { negaciones. }\end{array}$ & $\begin{array}{l}\text { Codificación de mensajes } \\
\text { complejos. } \\
\text { Recuperación de la } \\
\text { memoria de conocimientos } \\
\text { declarativos y } \\
\text { procedimentales. }\end{array}$ \\
\hline
\end{tabular}

Fig. 7. Programación neurocognitiva de interacción social. 


\section{Conclusiones}

La perspectiva didáctica tradicional se manifiesta como un marco insuficiente para evolucionar en el desarrollo de teorias metodológicas de aprendizaje de la L2 hacia nuevas propuestas eficientes para la adquisición de la L2 en el aula, descontextualizado de entornos auténticos. Se plantea la necesidad de superar el modelo actual que establece la comunicación como objetivo y las metodologías activas como dinámica constructivista en el aula sin considerar los factores neurobiológicos y neurocognitivos relevantes en el proceso de aprendizaje.

En este sentido se plantea como posibilidad la integración de conocimientos neurocientíficos determinantes del aprendizaje en la didactización de procesos de enseñanza/aprendizaje. Destacamos como determinantes los procesos atencionales, la memoria, y las emociones. En el contexto de la educación infantil se manifiesta como necesaria, además, la consideración del desarrollo cognitivo del niño para determinar la programación de objetivos de aprendizaje adecuados a su cronobiología cognitiva. Se plantea como objetivo principal potenciar la respuesta emocional positiva en el niño ante el proceso de aprendizaje dirigido en el aula para activar los procesos atencionales y así impulsar el reconocimiento de estímulos relevantes para el niño.

Este contexto de aprendizaje adecuado al cerebro debe quedar reflejado en el aprendizaje del alemán en el aula infantil en una propuesta de programación motivacional que considere la presentación multimodal en el aula del material lingüístico, la contextualización de la práctica lingüística en entornos cercanos o conectados con la realidad que perciben y con recursos metodológicos adecuados a su desarrollo psicopedagógico. La consideración de estos factores determina el diseño de actuaciones docentes que parten no solo de objetivos comunicativos y lingüísticos, sino también de actividades que integren la adquisición de la lengua alemana en procesos neurocognitivos auténticos, emocionalmente relevantes y que contribuyan a aunar la adquisición de la lengua alemana y el desarrollo neurocognitivo del niño.

En el marco concreto de la enseñanza de DaF la consecuencia más importante de nuestro planteamiento didáctico es que la progresión cíclica de confrontación con conocimientos tanto léxicos como gramaticales de la lengua alemana estarian organizados según criterios motivacionales y no meramente conceptuales. En este sentido, la aportación más relevante de la neurociencia es la evidencia de que sin los procesos atencionales activados de forma adecuada, no es posible el aprendizaje significativo necesario para adquirir nociones complejas como las correspondientes en lengua alemana a la declinación, la voz pasiva o la hipotaxis, en el plano gramatical, o la adquisición de un corpus léxico de máxima dificultad por las divergencias en la asignación de géneros y formas de plural. La adquisición de estas subcompetencias necesarias para la competencia comunicativa no solo se debería producir en situaciones comunicativas didactizadas en un método como corresponde a la metodología tradicional, sino mediante la secuenciación de recursos motivacionales asociadas a cada situación comunicativa, con el propósito de generar desde el punto de vista neurocognitivo las reacciones neurobiológicas adecuadas al aprendizaje. Una vez conseguida la activación adecuada de los procesos atencionales, la interacción con la lengua a nivel gramatical y léxico, por ejemplo, debería apoyarse en evidencias del funcionamiento de la memoria para la asignación y memorización de géneros, por ejemplo. Por otra parte, la presentación de nociones complejas, como las gramaticales, debería considerar algunas cuestiones como la relevancia de la presentación de patrones y estructuración ordenada de los procesos procedimentales en la generación de reglas. Debemos considerar que no es objetivo la superación de recursos metodológicos tradicionales, sino la optimización de los mis- 
mos mediante la secuenciación de recursos motivacionales como punto de partida, y la presentación de las reglas y la confrontación con el material lingüistico de forma adecuada al cerebro.

\section{REFERENCIAS BIBLIOGRÁFICAS}

ALBERCA, E., BORRUECO, M. (2018). Aprendizaje: el cerebro en el aula. En Borrueco, M. (Ed.). Ciencia e innovación docente en el aprendizaje de lenguas extranjeras (pp. 30-41). Sevilla: Egregius.

ÁLVAREZ, L., GONZÁLEZ-CASTRO, P., NÚÑEZ, J., GONZÁLEZ-PINEDA， J., ÁLVAREZ, D. y BERNARDO, A. (2007). Desarrollo de los procesos atencionales mediante actividades adaptadas. Papeles del Psicólogo, 28 (3), 211-217.

ANSARI, D., COCH, D., DE SMEDT B. (2010). Connecting education and cognitive neuroscience: where will the journey take us? Educational Philosophy and Theory, 43 (1-2), 1-7.

AXMACHER, N., HAUPT, S., COHEN, M. X., ELGER, C. E., FELL, J. (2009). Interference of working memory load with long-term memory formation. European Journal of Neuroscience, 29(7), 1501-1513.

BARERRA, M. L., DONOLO, D. (2009). Neurociencias y su importancia en contextos de aprendizaje. Revista Digital Universitaria, 10 (4), 1-18.

BERMEJO, V. (1987). Procesos atencionales y aplicaciones educativas. Psiquis, 331/87, $\mathrm{VIII/87,} \mathrm{46-53.}$

BORRUECO M., ALBERCA E. (2018). Neurodidáctica: Un camino para integrar los procesos neurocognitivos en los fundamentos de enseñanza/aprendizaje. En Borrueco, M. (Ed.). Ciencia e innovación docente en el aprendizaje de lenguas extranjeras (pp. 15-30). Sevilla: Egregius,

BOWERS, J. (2016). The practical and principled problems with educational neuroscience. Psychological Review. Advance online pu- blication. Consultado 25 de junio de 2019 http://dx.dol.org/10.1037/rev0000025.

BRANSFORD, J.D., BROWN, A.L., COCKING, R.R. (Ed.) (1999). How People Learn. Brain, Mind, Experience, and School. Washington: National Academy Press.

BUSCHMAN, T. J., KASTNER, S. (2015). From behavior to neural dynamics: an integrated theory of attention. Neuron, 88(1), 127144.

CANTÓ, J. (2015). Resultados de la implementación de la neurodidáctica en las aulas de educación infantil. Opcion, 31(5), 189199.

COSTA, A., SEBASTIÁN-GALLÉS, N. (2014). How does the bilingual experience sculpt the brain? Nature Reviews Neuroscience, 15, 336-345.

CHATTERJEE, N. (2016). Educational neuroscience: Challenges and Opportunities. Annals of neurosciences, 23, 63-65.

DAMASIO, A. R. (1998). Emotion in the perspective of an integrated nervous system. Brain Research Reviews, 26 (2-3), 83-86.

DÍAZ-CORRALEJO, J. (2003). Marco Común Europeo de Referencia en el aprendizaje de lenguas extranjeras. Revista de investigación e innovación en la clase de idiomas, 13-14, 32-43.

EICHENBAUM, H., NEAL J. C. (2001). From Conditioning to Conscious Recollection: Memory Systems of the Brain. Nueva York: Oxford University Press.

ELLIS, N. C. (1998). Emergentism, connectionism, and language learning. Language Learning, 48, 631-664.

ERTMER, P., NEWBY, T. (1993), Conductismo, cognitivismo y constructivismo: una com- 
paración de los aspectos críticos desde la perspectiva del diseño de instrucción. Performance Improvement Quarterly, 6(4), 50-72.

FERNÁNDEZ MARCH, A. (2006). Metodologias activas para la formación de competencias. Educatio siglo XXI, 24, 35-36.

FERNÁNDEZ, P. (2009). La influencia de las teorías psicolingüísticas en la didáctica de lenguas extranjeras: reflexiones en torno a la enseñanza del español L2. MarcoELE, 9, 1-33.

FIGUERAS, N. (2005). El Marco común europeo de referencia para las lenguas: de la teoría a la práctica. Carabela, 57, 5-23.

GARCÍA, A. (2012). Aportes de la evidencia neurológica para la lingüistica cognitiva. En GARCÍA, A., ORELLANO V., JAICHENCO V.; WAINSELBOIM, A. (Eds.). Lenguaje, cognición y cerebro (pp. 215-221). Mendoza: Editorial FFyL-UNCuyo y SAL.

GOLEMAN, D. (1996). La inteligencia emocional. Por qué es más importante que el coeficiente intelectual. México: Vergara Edición. JOHNSON J.S, NEWPORT E.L (1989). Critical period effects in second language learning: the influence of maturational state on the acquisition of English as a second language. Cognit Psycho/; 21: 60-99

KANDEL, E. R., HAWKINS, R. D. (1992). The biological basis of learning and individuality. Scientific American, 267(3), 78-87.

KANHEMAN, D. (1973). Attention and Effort. Englewood Cliffs, NJ: Prentice-Hall.

KLEIN, S. (2019). Learning. Principles and Applications. 8th Edition. California: SAGE.

KUHL, P., STEVENS, E., HAYASHI, A. DEGUCHI T., KIRITANI, SH. y IVERSON, P. (2006). Infants show a facilitation effect for native language phonetic perception between 6 and 12 months. Development Science 9(2), 13-21.

KUHL, P. (2010). Brain mechanism in early language acquisition. Neuron, 67, 713-727.
LANAUZE, M., SNOW, C. (1989). The relation between first and second language writing skills: Evidence from Puerto Rican elementary school children. Linguistics and education, 1(4), 323-339.

LEDESMA, M. A. (2015). Del conductismo, cognitivismo y constructivismo al conectivismo para la educación. Quito: Editorial Jurídica del Ecuador.

MARINA, J.A. (2014). Inteligencia ejecutiva. Madrid: Editorial Narcea.

MORA, F. (2013). Neuroeducación. Solo se puede aprender aquello que se ama. Madrid: Alianza Editorial.

MORGAN-SHORT, K., STEINHAUER, K., SANZ CH. y ULLMAN, M. (2012). Explicit and implicit second language training differentially affect the achievment of native-like brain activation patterns. J Cogn Neurosci, 24(4), 933-947.

NÚÑEZ, P., MORAL C. (2010). Competencia léxica y competencia comunicativa: bases para el diseño de programas didácticos en la educación escolar. Lenguaje y textos, 23, 91-97.

PANIAGUA, M. N. (2016). Marcadores del desarrollo infantil: enfoque neuropsicopedagógico. Fides et ratio, 12, 81-99.

PAPATZIKIS E. (2017). Neuromyths in education and development: A comprehensive approach. European Scientific Journal, Special edition, 85-91.

REICH, K. (1998). Konstruktivistische Unterrichtsmethoden. System Schule, 2(1), 20-26.

RIZZOLATI, G. (2005). The mirror neuron system and its function in humans. Anatomy and embryology, 210(5-6), 419-421.

ROTH, T. (2011). Neurodidaktik - Chancen und Grenzen am Beispiel Accelerated Learning. Über die Brizanz einer interdisziplinären Zusammenarbeit von Neurobiologie und Pädagogik. Saarbrücken: VDM Verlag.

RUEDA R., POSNER M., ROTHBART M. (2005). The Development of Executive Atten- 
tion. Contributions to the Emergence of Self-Regulation, Developmental Neuropsychology, 28(2), 57-594.

RUIZ-CONTRERAS, A. y CANSINO, S. (2005). La atención y la memoria episódica: revisión de estudios en modalidad visual. Revista Neurología, 41(12), 733-743.

SALCEDO, P. (2018). Neuroeducación: Incorporación de enseñanza basada en el funcionamiento del cerebro. WBT en la metodología CLIL. Publicaciones Didácticas, 90 (1), 397-430.

SÁNCHEZ -REYES, M.S. (2000). La influencia en la edad temprana en el proceso de adquisición de la lengua extranjera. Aula, 12, 43-53.

SÁNCHEZ, A. (2009). La enseñanza de idiomas en los últimos cien años: métodos y enfoques. Murcia: SGEL.

SCHACTER, D.L., TULVING, E. (1994). What are the memory systems of 1994? En Schacter, D.L., Tulving, E. (Eds). Memory Systems (pp.1-38). Cambridge: MIT Press 1-38.
TERIGI, F. (2016). Sobre aprendizaje escolar y neurociencia. Propuesta educativa, 46, 50-64.

TEXTOR, M.R. (2010). Gehirnentwicklung im Kleinkindalter - Konsequenzen für die frühkindliche Bildung. Consultado el 25 de junio de 2019, https://kindergartenpaedagogik.de/fachartikel/psychologie/779

TORRES, J. R. (2005). El mito del periodo crítico para el aprendizaje de la pronunciación de un idioma extranjero. PHONICA, I, 1-9.

TULVING, E. (1985). How many memory systems are there? American Psychologist, 40(4), 385.

ULLMAN, M T. (2004). Contributions of memory circuits to language: The declarative/procedural model. Cognition, 92: 231-270.

VARELA, F., THOMPSON, E., ROSCH, E. (1991). The Embodied Mind. Cambridge: MIT Press.

WOLFE, P. (2001). Translating Research into Classroom Practice. USA, Virginia: ASCD. 
